\title{
Isolation and High Throughput Flow Cytometric Apoptosis Assay of Human Neutrophils to Enable Compound Library Screening
}

\author{
K. D. Herman ${ }^{1}$, A. Rahman ${ }^{2}$ and L. R. Prince ${ }^{1, *}$
}

\begin{abstract}
${ }^{1}$ Department of Infection, Immunity and Cardiovascular Disease, University of Sheffield, Sheffield, United Kingdom; '2Department of Biochemistry and Molecular Biology, Faculty of Biological Sciences, University of Dhaka, Dhaka, Bangladesh

*For correspondence: L.r.prince@sheffield.ac.uk
\end{abstract}

[Abstract] The study of human neutrophils in vitro is challenging due to their short half-life and propensity for activation. However, with careful handling and manipulation in the laboratory, they can be a powerful tool to investigate immune responses in health and disease. Here we describe a method for the isolation of human neutrophils from peripheral blood samples, followed by a high-throughput screen to assess the efficacy of a library of compounds in inducing neutrophil apoptosis, which may have therapeutic potential in neutrophil-driven diseases. This protocol is based on previously-published neutrophil isolation methods utilizing Dextran sedimentation of red blood cells followed by the separation of granulocytes with plasma/Percoll discontinuous gradient centrifugation. Yields of $\sim 1 \times 10^{6}$ neutrophils per millilitre of blood, and purities of $>95 \%$ neutrophils are typical. Neutrophils are treated with a library of kinase inhibitors, followed by flow cytometry to assess the rate of neutrophil apoptosis. This protocol allows for the high-throughput screening of primary human immune cells to identify compounds with a potential to modify neutrophil function, and could be modified to assess other phenotypes if required.

Keywords: Neutrophil, Apoptosis, Flow cytometry, Kinase, Screen, Compound library

[Background] Neutrophils are important innate immune cells with keys roles in defense against infection. They are short-lived cells with a typical half-life in the circulation of 6-8h which is extended at sites of infection and inflammation (Summers et al., 2010; Hidalgo et al., 2019). Although this allows neutrophils to complete critical effector functions, a failure to undergo programmed cell death (apoptosis) leads to the release of neutrophil contents that are histotoxic and ultimately perpetuates tissue damage and inflammation. Neutrophil apoptosis is anti-inflammatory, both by removing effete neutrophils from the tissue and promotion of their clearance by macrophages (efferocytosis). The precise regulation of neutrophil apoptosis is therefore critical and if therapeutically targeted may provide a route into novel medicines for inflammatory diseases in which neutrophils predominate. A number of studies highlight the importance of protein kinases in regulating neutrophil apoptosis (Webb et al., 2000; Rossi et al., 2006; Burgon et al., 2014) and a number of small molecule inhibitors are in development (Wu et al., 2015). Here we have screened a library of kinase inhibitors in a high-throughput flow cytometric assay to identify novel regulators of neutrophil apoptosis.

In this protocol, neutrophils are isolated from human blood by dextran sedimentation and discontinuous plasma/Percoll gradient centrifugation, based on previous protocols (Haslett et al., 1985). 
Neutrophils are extremely sensitive to activation, particularly with respect to rates of cell death, so minor modifications have been made in order to minimize handling and activation of cells. We have developed a flow cytometric assay using the Attune Autosampler to allow us to screen compound libraries in a fast and efficient way, using Annexin- $V$ and TOPRO-3 as markers of apoptosis. Testing up to 88 compounds in a single experiment minimizes prep-to-prep variation, including technical and donor variation and allows experimental repeats to be completed in a timely manner.

\section{Materials and Reagents}

1. Petri dishes: $90 \mathrm{~mm}$ diameter $\times 15 \mathrm{~mm}$ deep (Thermo Fisher Scientific, catalog number: 101R20)

2. $15 \mathrm{ml}$ polypropylene tubes (SARSTEDT, catalog number: 62.554 .502 )

3. $50 \mathrm{ml}$ polypropylene tubes (SARSTEDT, catalog number: 62.547 .254 )

4. $1.5 \mathrm{ml}$ centrifuge tubes

5. $0.2 \mu \mathrm{m}$ syringe filter

6. Transfer pipette $3.5 \mathrm{ml}$ (SARSTEDT, catalog number: 86.1171 .001$)$

7. 96-well flexible, untreated polyvinyl chloride general assay plates (Corning, Sigma-Aldrich, catalog number: CLS2595)

8. 96-well firm flat bottom transparent plates (Attune autosampler compatible, e.g., Nunc via Invitrogen)

9. BD Plastipak Eccentric Luer-Slip Syringe (Fisher Scientific, catalog number: 300866)

10. Serological pipettes (Fisher Scientific, catalog number: 13-676-10J)

11. Tri-sodium Citrate BP $3.8 \%$ w/v (Martindale Pharmaceuticals, catalog number: MS156)

12. Dextran T500 (Pharmacosmos, catalog number: 5510-0500-9007)

13. $0.9 \%$ Saline (sodium chloride) (Baxter Healthcare Corporation, catalog number: UKF124)

14. Percoll (GE Healthcare, catalog number: 17-0891-02)

15. RPMI 1640 media (Lonza, catalog number: BE12-702F)

16. Hank's Balanced Salt Solution (Gibco, Life Technologies, catalog number: 14170-112)

17. Fetal Bovine Serum (FBS) (Sigma-Aldrich, catalog number: F2442)

18. Penicillin-streptomycin (Gibco, Life Technologies, catalog number: 15140122)

19. 1x Phosphate Buffered Saline (Gibco, Life Technologies, catalog number: 10010023)

20. Recombinant Human Granulocyte-Macrophage Colony-Stimulating Factor (GMCSF) (PeproTech, catalog number: 300-03)

21. Pyocyanin (prepared as previously described (Usher et al., 2002) and now commercially available from a number of suppliers)

22. Annexin binding buffer (ABB) (BD Pharmingen, BD Biosciences, catalog number: 556454)

23. Dimethyl sulfoxide (DMSO) (Sigma-Aldrich, catalog number: D8418)

24. PE-conjugated annexin-V (BD Pharmingen, BD Biosciences, catalog number: 556421)

25. TOPRO-3 (Molecular Probes, Life Technologies, catalog number: T3605)

26. Published kinase inhibitor set (PKIS) (commercially available (Elkins et al., 2016) 
27. EDTA (Sigma-Aldrich, catalog number: E6758)

28. Ethanol absolute (Fisher Scientific, catalog number: 10428671)

29. Kwik-Diff Solution 2 (Thermo Fisher Scientific, catalog number: 9990706)

30. Kwik-Diff Solution 3 (Thermo Fisher Scientific, catalog number: 9990707)

31. $6 \%$ Dextran solution (see Recipes)

32. $90 \%$ Percoll (see Recipes)

33. $70 \%$ Ethanol solution (see Recipes)

34. Media (see Recipes)

35. Annexin binding buffer (ABB) (see Recipes)

36. PE-conjugated Annexin-V (1:20 dilution) (see Recipes)

37. TOPRO-3 (1:7,500 dilution) (see Recipes)

\section{Equipment}

1. BD Vacutainer Safety-Lok blood collection set (Becton, Dickinson and Company, catalog number: 367282)

2. Water bath (NE1-8, Nickel-Electro Ltd, Weston-super-Mare, UK)

3. Class II Microbiological Safety cabinet (Walker Safety Cabinets, catalog number: 50-600-467)

4. Multi-channel pipette (Alpha Laboratories, catalog number: AP80300)

5. Heraeus Biofuge Fresco (Thermo Fisher Scientific, catalog number: 75005521)

6. Large capacity centrifuge (DBJ Labcare Ltd., model: MSE Mistral 3000i)

7. $\mathrm{CO}_{2}$ incubator (Sanyo Electric Co Ltd., model: MCO-17AIC)

8. Cell counting chamber (VWR, catalog number: 630-1510)

9. Attune flow cytometer (Attune, Applied Biosystems, Life Technologies, Paisley, UK)

10. Cytospin 4 cytocentrifuge (Thermo Fisher Scientific, catalog number: A78300003)

\section{Software}

1. FlowJo flow cytometric data analysis software (FlowJo, LLC)

2. GraphPad Prism 8 (GraphPad Software, San Diego, CA)

\section{Procedure}

\section{Part I: Isolation of neutrophils from peripheral blood}

Blood was obtained from healthy subjects following approval from the local ethics committee and informed consent from volunteers. All scientists handling blood and blood products were vaccinated against hepatitis B. Aside from the blood collection and centrifugation steps, carry out all work inside a Class II Biological Safety Cabinet. 


\section{A. Preparation}

1. Prepare a Class II Biological Safety Cabinet for use by cleaning with $70 \%$ ethanol or similar.

2. Pre-warm the $6 \%$ Dextran solution in a water bath set to $37^{\circ} \mathrm{C}$.

3. Place the $90 \%$ Percoll solution into the cabinet to allow it to reach room temperature.

4. Prepare a $50 \mathrm{ml}$ tube for blood collection by adding $5 \mathrm{ml} 3.8 \%$ tri-sodium citrate as an anticoagulant.

Note: Concentration of tri-sodium citrate $=11 \%$ total volume .

B. Blood collection

1. Obtain peripheral whole blood samples by venepuncture using a BD Vacutainer Safety-Lok blood collection set, collecting $40 \mathrm{ml}$ of blood into a $50 \mathrm{ml}$ syringe.

Note: Blood is typically obtained at the same time of day (between 8-10 am).

2. Immediately transfer the blood into the $50 \mathrm{ml}$ tube containing tri-sodium citrate, running the blood slowly down the side of the tube to avoid the formation of bubbles. Invert slowly several times to mix.

C. Neutrophil isolation

1. Centrifuge the blood at $323 \times g$ at $20^{\circ} \mathrm{C}$ for 20 min (Figure 1, Spin 1).

This results in the separation of the blood sample into two distinct layers of platelet-rich plasma (PRP) and blood cells (Figure 1A).

2. Using a serological pipette followed by a Pasteur pipette, transfer the PRP to a fresh $50 \mathrm{ml}$ tube. Avoid disturbing the interface.

3. Centrifuge the PRP at $896 \times g$ for $20 \mathrm{~min}$ at $20{ }^{\circ} \mathrm{C}$ (Figure 1, Spin 2) to pellet the platelets. Following centrifugation of the PRP, transfer the supernatant (PPP, Figure 1B) to a fresh $50 \mathrm{ml}$ tube. The platelet pellet can be discarded.

4. During this centrifugation step, add $6 \mathrm{ml}$ of the pre-warmed $6 \%$ dextran solution to the remaining blood cell layer from Step C1, and top up to $50 \mathrm{ml}$ with saline. Replace the lid and mix gently by inversion. Take off the lid and use a Pasteur pipette to remove any bubbles that have formed around the top of the tube or in the lid.

5. Loosely replace the lid, and leave undisturbed at room temperature for 20-30 min. A clear interface should be visible.

6. Following dextran sedimentation (Figure 1C), transfer the pale upper leukocyte layer to a clean $50 \mathrm{ml}$ tube using a serological pipette followed by a Pasteur pipette. Take care to not to disturb the interface.

7. Centrifuge at $224 \times g$ at $20^{\circ} \mathrm{C}$ for 6 min (Figure 1, Spin 3).

8. During the centrifugation step, the discontinuous plasma/Percoll gradient is prepared.

a. Label two separate $15 \mathrm{ml}$ tubes as "upper phase" and "lower phase".

b. To make the lower phase, add $1.02 \mathrm{ml}$ of $90 \%$ Percoll (Recipe 2) and $0.98 \mathrm{ml}$ PPP. Mix carefully by pipetting up and down, avoiding bubbles. 
Note: For accuracy use a 1000 pipette to make the gradient layers.

c. To make the upper phase, combine $0.84 \mathrm{ml} 90 \%$ Percoll with $1.16 \mathrm{ml}$ PPP. Mix carefully as above.

d. Using a Pasteur pipette, take up all $2 \mathrm{ml}$ of the upper phase and very slowly deposit it on top of the lower phase. Hold the tube containing the lower phase at a $45^{\circ}$ angle so the liquid slowly runs down the side of the tube and sits on top of the lower phase.

Note: Avoid the formation of bubbles.

9. Once the centrifugation (Step C7) is complete, remove and discard the supernatant, and gently resuspend the leukocyte pellet in $2 \mathrm{ml}$ PPP by gently swirling the tube in a circular motion.

Note: The cell pellet is soft and extra care is required when handling.

10. Deposit all $2 \mathrm{ml}$ of the resuspended leukocyte population on top of the upper phase of the gradient using the same careful technique as in Step C8d (Figure 1D).

11. Centrifuge the tube containing the gradient at $271 \times \mathrm{g}$ for $11 \mathrm{~min}$ at $20^{\circ} \mathrm{C}$ with no brake (Figure 1, Spin 4).

Note: This yields three layers of cells: remaining red blood cells at the bottom of the tube, granulocytes in the middle, and peripheral blood mononuclear cells (PBMCs) closest to the top (Figure 1E).

12. Using a Pasteur pipette, remove the PBMC layer into a clean $50 \mathrm{ml}$ tube, being sure to remove as many cells as possible as residual PBMCs may contaminate the granulocyte layer.

Note: PBMCs can be resuspended in media and used in other protocols or discarded if not required.

13. Using a Pasteur pipette, remove the granulocyte layer into a clean $50 \mathrm{ml}$ tube, taking as many cells as possible but avoid disturbing the red blood cell pellet.

14. Add $10 \mathrm{ml} \mathrm{PPP}$ to the tube, top up to $40 \mathrm{ml}$ with Hank's Balanced Salt Solution (HBSS) and mix by inversion to ensure an even cell suspension.

15. Count cells using a cell counting chamber.

16. Centrifuge at $504 \times g$ for 6 min at $20^{\circ} \mathrm{C}$ (Figure 1, Spin 5).

17. Remove the supernatant and gently resuspend the cell pellet in media (Recipe 4) to a final concentration of $5 \times 10^{6}$ cells $/ \mathrm{ml}$.

18. Freshly isolated neutrophils are cytocentrifuged ( $300 \mathrm{rpm}, 3 \mathrm{~min}$, Cytospin 4 cytocentrifuge) onto microscope slides and stained with Kwik-Diff dyes. The purity of the prep is determined by assessment of cell morphology (Figure 2A). These slides can also be used to identify apoptotic neutrophils, based on morphological changes (Figure 2B).

Note: Neutrophils that have been in culture are centrifuged at $200 \mathrm{rpm}, 3 \mathrm{~min}$. 
A
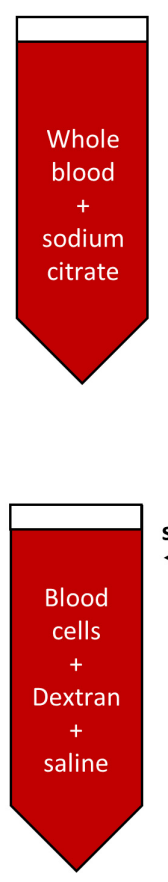
saline to blood cells
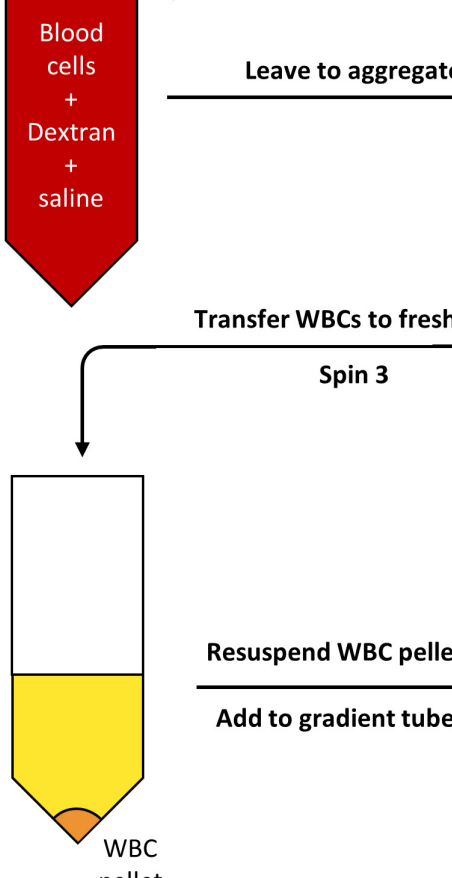

Spin 3

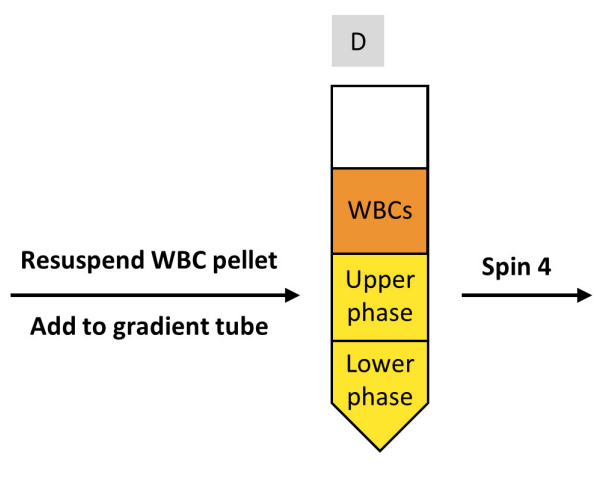

E

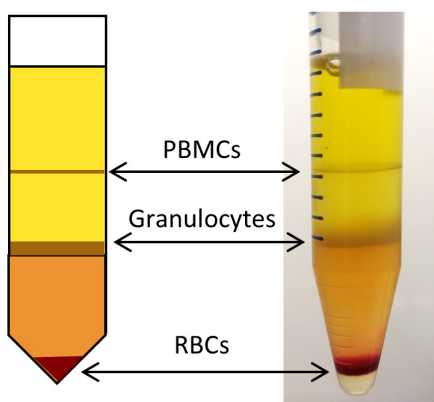

Transfer PBMCs and granulocyte layers to fresh tubes

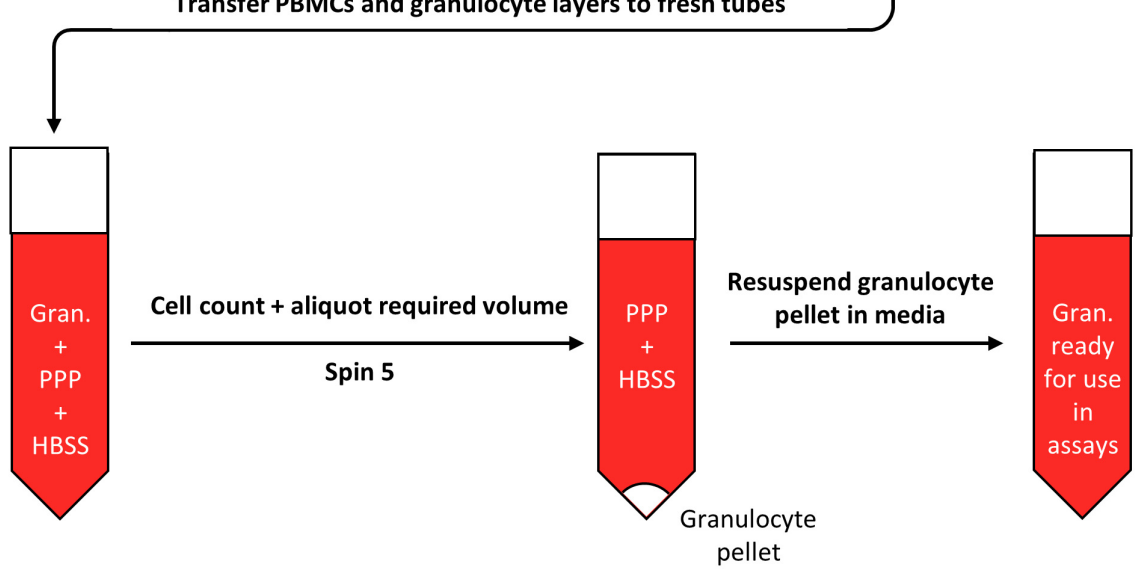

Figure 1. Flow diagram of the neutrophil isolation protocol 


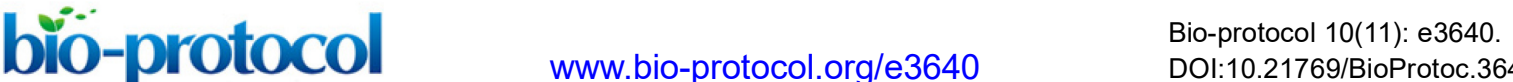

A)

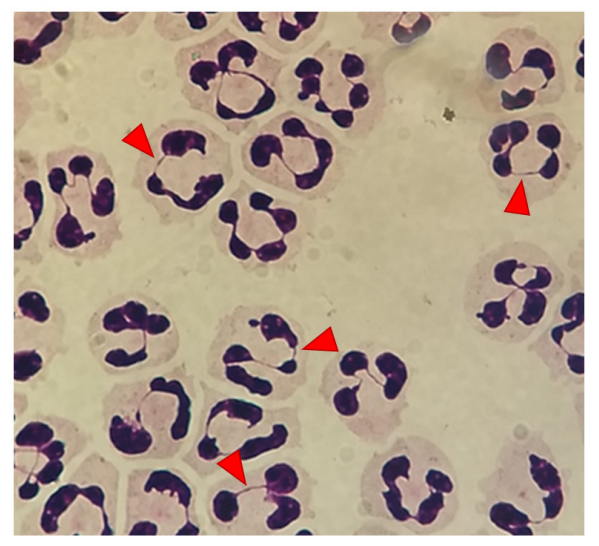

B)

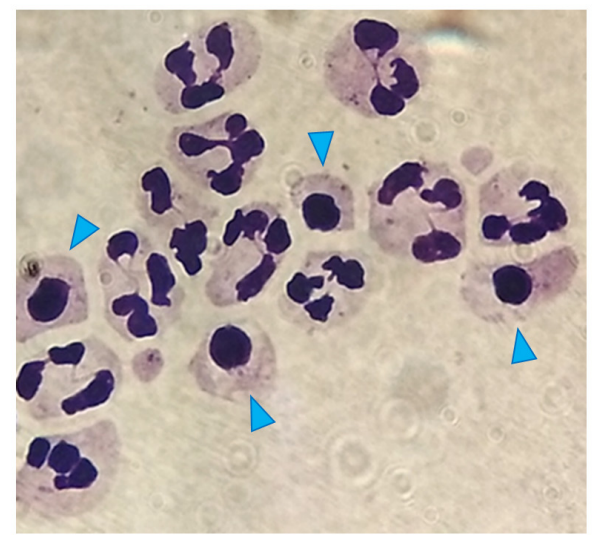

Figure 2. Healthy and apoptotic neutrophil morphology. Immediately after isolation (A) neutrophils are healthy, with lobes of the nucleus connected by string-like chromatin bridges (red arrows). Apoptotic neutrophils (B, blue arrows) are identifiable by their rounded-up nuclei and lack of chromatin bridges. Visualised on Kwik-Diff stained cytocentrifuge slides by oil immersion microscopy.

\section{Part II: High-throughput kinase inhibitor apoptosis assay}

Note: See Figure 3 for a schematic summary of this section of the protocol. 


\section{Step Protocol summary Contents of each well}

A1-3

Make sub-stocks of all compounds/controls in media

Plate A:

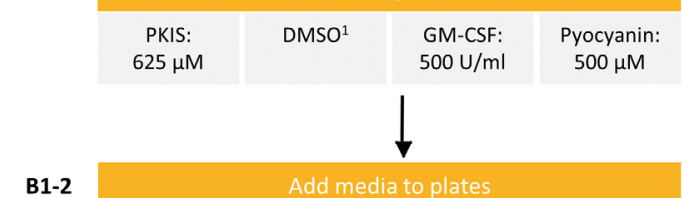

B1-2
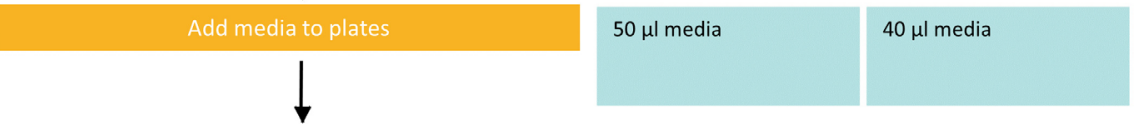

B3

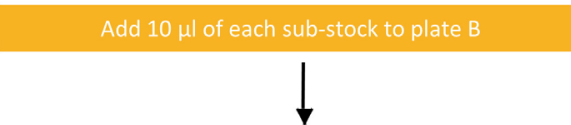

B4-6
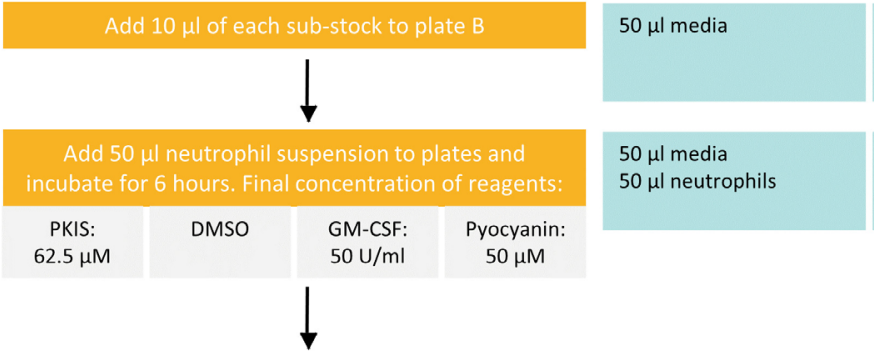

$40 \mu \mathrm{l}$ media

$50 \mu \mathrm{lmedia}$

$10 \mu \mathrm{l}$ compound/control

C1-4

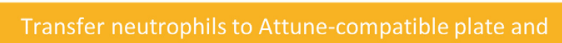

wash with PBS
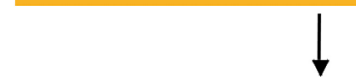

Neutrophils

Neutrophils

C5

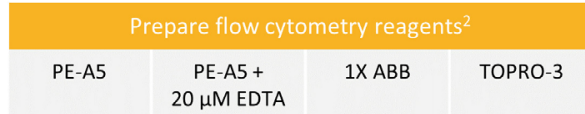

$50 \mu$ l neutrophils

$40 \mu \mathrm{l}$ media

$10 \mu \mathrm{l}$ compound/control

$50 \mu$ neutrophils

66-7

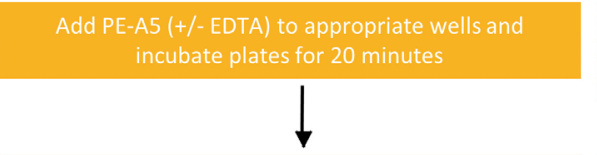

Neutrophils

$50 \mu \mathrm{IPE}-\mathrm{A} 5$ (+/-EDTA) ${ }^{3}$

Neutrophils

$50 \mu \mathrm{lPE}-\mathrm{A} 5$

C8-10
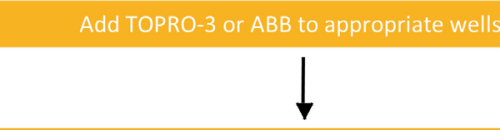

$\downarrow$

Neutrophils

$50 \mu \mathrm{IPE}-\mathrm{A} 5\left(+/-\mathrm{EDTA}^{3}\right.$

$150 \mu \mathrm{I}$ TOPRO-3/ABB ${ }^{3}$

C11

D1-3

Perform sample acquisition using an Attune Flow

${ }^{1}$ Equivalent dilution ratio $(\mathrm{v} / \mathrm{v})$ as the other control reagents

${ }^{2}$ See Recipes

${ }^{3}$ See Table 3 for specific wells requiring EDTA

\section{Figure 3. Flow diagram of the apoptosis screening protocol}

A. Preparation of test compounds

1. Published Kinase Inhibitor Set (PKIS) compounds dissolved in DMSO come pre-aliquoted into 96-well plates. Using a multi-channel pipette, transfer an aliquot of each compound to a clean 96-well plate and dilute in media to achieve a 10x final concentration sub-stock (in this case $625 \mu \mathrm{M})$.

2. In sterile Eppendorf tubes, prepare dilutions of control reagents (in this case pyocyanin and GMCSF, which accelerates and inhibits neutrophil apoptosis, respectively) to achieve 10x final 
concentration sub-stocks ( $500 \mu \mathrm{M}$ for pyocyanin and $500 \mathrm{U} / \mathrm{ml}$ for GM-CSF). Prepare a DMSO control by diluting DMSO in media at the same ratio $(\mathrm{v} / \mathrm{v})$ as the control reagents.

3. Keep all reagents on ice until required.

Note: Alternative inhibitor libraries and control compounds may be more appropriate for user assays.

B. Seeding plates

1. Take two sterile 96-well flexible, untreated polyvinyl chloride general assay plates and label the control plate: A and test plate: $\mathrm{B}$.

2. Add $50 \mu \mathrm{l}$ media to 8 wells of plate $A$ (see plate $A$ template below).

Note: Plate A includes wells for technical controls including unstained samples and single stain controls for Annexin-V and TOPRO-3. Since Annexin-V binding is a calcium dependent process, an additional control containing EDTA is included.

3. To plate $B$ add kinase inhibitor compounds [62.5 $\mu \mathrm{M}]$ and assay control compounds (media, DMSO (vehicle control), GMCSF [50 U/ml] and pyocyanin [50 $\mu \mathrm{M}]$ ) in corresponding duplicate wells (see plate B template). Using a multi-channel pipette add $10 \mu \mathrm{l}$ of each kinase inhibitor compound sub-stock (see Step A1) to wells containing $40 \mu \mathrm{l}$ of media. Add $10 \mu \mathrm{l}$ each of pyocyanin, GM-CSF and DMSO sub-stock (see Step A2) to wells containing $40 \mu \mathrm{l}$ of media. Include a final 2 wells containing $50 \mu$ of media.

4. Resuspend $30 \times 10^{6}$ freshly isolated neutrophils in $6 \mathrm{ml}$ of warm media at a final density of $5 \times$ $10^{6} / \mathrm{ml}$ (see Step C17 of Part I).

5. Transfer the cell suspension to a sterile Petri dish and using a multichannel pipette, transfer $50 \mu \mathrm{l}$ of the cell suspension to each well of the 8 wells of plate $A$ and all wells of plate $B$.

6. Incubate in a humified incubator at $37^{\circ} \mathrm{C}$ and $5 \% \mathrm{CO}_{2}$ for $6 \mathrm{~h}$.

Note: Concentrations of inhibitors and control compounds are calculated based on a final volume of $100 \mu \mathrm{l}$ per well, since $50 \mu \mathrm{l}$ neutrophil suspension is added making a total volume of $100 \mu \mathrm{l}$ (Figure 3). Each well contains $2.5 \times 10^{5}$ cells.

See suggested templates for staining control and assay plates below (Tables 1-2):

Table 1. Plate A template (flow cytometric controls)

\begin{tabular}{|c|c|c|c|c|c|c|c|c|c|c|c|c|}
\hline & 1 & 2 & 3 & 4 & 5 & 6 & 7 & 8 & 9 & 10 & 11 & 12 \\
\hline A & $U$ & $U$ & A5 & A5 & EDTA & EDTA & T3 & T3 & & & & \\
\hline B & & & & & & & & & & & & \\
\hline C & & & & & & & & & & & & \\
\hline$D$ & & & & & & & & & & & & \\
\hline$E$ & & & & & & & & & & & & \\
\hline $\mathrm{F}$ & & & & & & & & & & & & \\
\hline G & & & & & & & & & & & & \\
\hline $\mathrm{H}$ & & & & & & & & & & & & \\
\hline
\end{tabular}


Table 2. Plate B template (test plate)

\begin{tabular}{|c|c|c|c|c|c|c|c|c|c|c|c|c|}
\hline & 1 & 2 & 3 & 4 & 5 & 6 & 7 & 8 & 9 & 10 & 11 & 12 \\
\hline$A$ & $M$ & $\mathrm{M}$ & $D$ & $\mathrm{D}$ & $\mathrm{G}$ & $G$ & $P$ & $P$ & 1 & 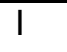 & 1 & $\mathrm{I}$ \\
\hline B & I & I & I & I & I & I & I & I & I & I & I & I \\
\hline C & I & I & I & I & I & I & I & I & I & I & I & I \\
\hline$D$ & I & I & I & I & I & I & I & I & I & I & I & I \\
\hline$E$ & I & I & I & I & I & I & I & I & I & I & I & 1 \\
\hline$F$ & I & I & I & I & I & I & I & I & 1 & I & I & I \\
\hline $\mathrm{G}$ & I & I & I & I & I & I & I & I & I & I & I & I \\
\hline $\mathrm{H}$ & I & I & I & I & I & I & I & I & I & I & I & I \\
\hline
\end{tabular}

M = Media; D = DMSO; G = GMCSF; $\mathrm{P}$ = Pyocyanin and I = Inhibitor compounds

C. Staining cells for flow cytometric apoptosis assay

1. Following the incubation period, use a multi-channel pipette to resuspend the cells by gently pipetting up and down 2-3 times and transfer the cell suspension to Attune autosampler compatible, firm 96-well plates. Keep the plate on ice.

2. Centrifuge the plates at $378 \times g$ for 6 min at $4{ }^{\circ} \mathrm{C}$.

3. Using a multi-channel pipette, remove supernatants carefully without disturbing the cells and discard the supernatants.

4. Using a multi-channel pipette, add $150 \mu \mathrm{l}$ ice cold PBS, gently resuspend cells to wash and centrifuge plates as above.

5. During the centrifugation prepare the following:

1x ABB (Annexin Binding Buffer) by diluting 10x ABB (see Recipe 5)

PE-conjugated Annexin-V (see Recipe 6)

6. Following centrifugation, remove supernatants and immediately add $50 \mu$ diluted PEconjugated annexin- $V$ to all wells except unstained and TOPRO-3 single stain control wells (see Table 3 below) and mix gently. To EDTA control wells add EDTA to a final concentration of $0.02 \mathrm{M}$.

Table 3. Cell death probe staining conditions

\begin{tabular}{lll}
\hline Conditions & PE-Annexin V & TOPRO-3 \\
\hline Media (M) & + & + \\
DMSO (D) & + & + \\
GMCSF (G) (anti-apoptotic) & + & + \\
Pyocyanin (P) (pro-apoptotic) & + & + \\
Inhibitor compounds (I) & + & - \\
Unstained (U) & - & - \\
Annexin V single stain control & + & - \\
EDTA control & + & + \\
TOPRO-3 single stain control & - &
\end{tabular}


7. Cover the plate with aluminium foil to minimise exposure to light and incubate for $20 \mathrm{~min}$ at room temperature in the dark.

8. During the incubation period, prepare TOPRO-3 solution (1:7,500 v/v) in 1x ABB (see Recipe 7).

9. Following the incubation period, add $150 \mu \mathrm{l}$ of the TOPRO-3 solution to all wells except unstained, annexin- $\mathrm{V}$ single stain and EDTA control wells (see Table 3 above).

10. To each of the unstained, annexin- $V$ single stain and EDTA control wells, add $150 \mu \mathrm{l}$ of $1 \times$ ABB.

11. Transfer samples from wells of plate $A$ to corresponding $1.5 \mathrm{ml}$ centrifuge tubes. The unstained and single strain controls in the tubes will be used to set up cytometer parameters during sample acquisition.

D. Flow cytometry sample acquisition

Perform sample acquisition with an Attune ${ }^{\mathrm{TM}}$ Flow Cytometer using Attune ${ }^{\mathrm{TM}}$ Cytometric Software as per suggested protocols.

1. Acquire sample from tubes for unstained and single stain controls in order to set up parameter settings.

2. Gate neutrophils based on typical forward (FSC) and side (SSC) scatter profiles (see Figure 4A) (Prince et al., 2012).

3. Perform sample acquisition using a built-in auto sampler, which acquires samples from 96 wells plate in a robotic manner based on a pre-set program. Set the flow cytometer to collect a predefined number (e.g., 10,000) of gated events from each well. The plate was routinely read from well $\mathrm{A} 1$ to $\mathrm{H} 12$.

Note: Each 96-well plate takes approximately $60 \mathrm{~min}$ to acquire therefore we recommend limiting the number of plates sampled in order to avoid lengthy acquisition times which may affect results.

E. Data analysis

1. Export data to .fcs files and analyse by flow cytometry analysis software FlowJo.

2. Gate neutrophils based typical forward (FSC) and side (SSC) scatter profiles (see Figure 4A).

3. Analyse the gated neutrophils based on the quadrant distribution of annexin- $V$ and TOPRO-3 positive events (see Figure 4B). Determine percentage of cells appearing in each quadrant.

4. Calculate percent apoptosis by the summation of annexin- $V$ positive and annexin-V/TOPRO-3 double positive events.

5. Calculate average percent apoptosis from at least three independent experiments.

6. Calculate apoptosis fold change for inhibitor compounds compared to DMSO controls. Note: In optimisation experiments we confirmed percentage apoptosis as determined by high throughput flow cytometry closely correlated with apoptosis measured by light microscopy. 
A

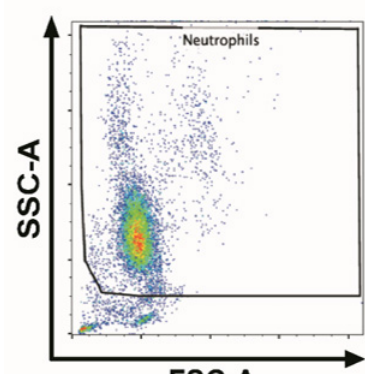

FSC-A
B

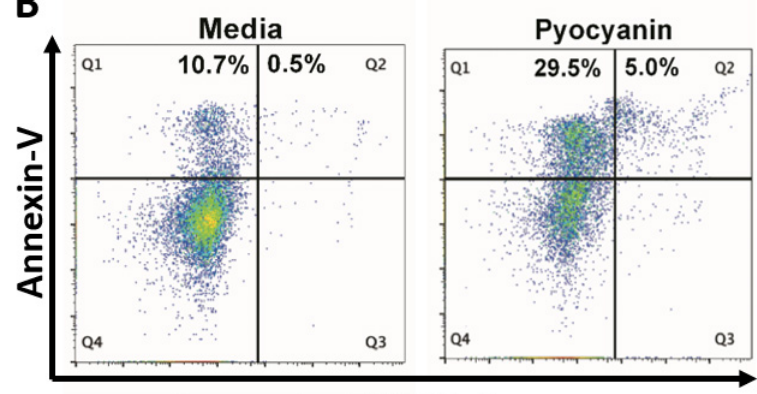

TOPRO-3

Figure 4. Flow cytometry gating strategy for the assessment of neutrophil apoptosis. A. Neutrophils were first gated based on the forward scatter (FSC-A) and side scatter (SSC-A) properties. The FSC-A versus SSC-A distribution is shown in a pseudo colour dot plot. (B) Gated neutrophils were then analysed based on the annexin-V versus TOPRO-3 distribution profile, and apoptosis calculated by the summation of annexin-V single positive (Q1) and annexin-V/ TOPRO-3 double positive (Q2) events. Representative examples showing the distribution of annexin-V/TOPRO-3 in media- and pyocyanin-treated neutrophils.

\section{Data analysis}

This protocol generally yields a leukocyte population containing over $95 \%$ neutrophils. Other cells present can include eosinophils, monocytes, lymphocytes and red blood cells. This is assessed in our experiments by cytocentrifugation and staining of the final cell population with Kwik-Diff stains 2 and 3, which allows for differential identification of blood cells. Isolations yielding neutrophils at a purity of < 95\% are not included. See original citation for further details (Rahman et al., 2019).

Each neutrophil isolation is carried out on a whole blood sample from a single donor, and data from each donor is considered as an independent experiment (i.e., $n=1$ ). Datasets are of a minimum of $n=3$ and mean \pm SEM is calculated. Statistical analysis was performed using GraphPad Prism 8 (GraphPad Software, San Diego, CA) and significance accepted at $P<0.05$. See original citation for further details (Rahman et al., 2019).

\section{Recipes}

1. $6 \%$ Dextran solution

$3 \mathrm{~g}$ Dextran

$50 \mathrm{ml}$ saline

Dissolve at $37^{\circ} \mathrm{C}$ in water bath and filter sterilize using a $50 \mathrm{ml}$ syringe and $0.2 \mu \mathrm{m}$ syringe filter. Once made, solution can be stored at $2-8^{\circ} \mathrm{C}$ for up to two weeks

2. $90 \%$ Percoll

$9 \mathrm{ml}$ Percoll stock

$1 \mathrm{ml}$ sterile saline 
3. $70 \%$ ethanol solution

Prepare $70 \%$ v/v ethanol solution with distilled water

4. Media

500 ml RPMI 1640

10\% Fetal Calf Serum (Heat-inactivated)

$1 \%$ Penicillin-Streptomycin

Keep the reconstituted media at $4{ }^{\circ} \mathrm{C}$, away from light

5. Annexin binding buffer $(\mathrm{ABB})(30 \mathrm{ml})$

$3 \mathrm{ml}$ 10x ABB

$27 \mathrm{ml}$ distilled water

Fresh preparation is recommended

6. PE-conjugated Annexin-V (1:20 dilution; enough for 100 wells)

$4.75 \mathrm{ml} \mathrm{ABB}(47.5 \times 100=4,750 \mu \mathrm{l})$

$250 \mu \mathrm{PE}$ conjugated Annexin- $\mathrm{V}$

7. TOPRO-3 (1:7,500 dilution)

$20 \mathrm{ml} \mathrm{ABB}$

$2.66 \mu$ I TOPRO-3

\section{Acknowledgments}

Commonwealth Scholarship Commission-funded PhD studentship for Atiqur Rahman. This protocol was adapted from previous work (Haslett et al., 1985).

\section{Competing interests}

The authors have no competing interests to declare.

\section{Ethics}

In vitro experiments in this study were conducted on neutrophils isolated from peripheral blood samples of healthy donors, in compliance with the guidelines of the South Sheffield Research Ethics Committee (for young healthy subjects; reference number STH13927). In addition, peripheral blood samples from patients with Chronic Obstructive Pulmonary Disease and age-matched healthy controls were also utilised, in compliance with the National Research Ethics Service (NRES) Committee Yorkshire and the Humber (reference number: 10/H1016/25). Informed consent was obtained from all donors after the nature and possible consequences of the study were explained. 


\section{References}

1. Burgon, J., Robertson, A. L., Sadiku, P., Wang, X., Hooper-Greenhill, E., Prince, L. R., Walker, P., Hoggett, E. E., Ward, J. R., Farrow, S. N., Zuercher, W. J., Jeffrey, P., Savage, C. O., Ingham, P. W., Hurlstone, A. F., Whyte, M. K. and Renshaw, S. A. (2014). Serum and glucocorticoidregulated kinase 1 regulates neutrophil clearance during inflammation resolution. $J$ Immunol 192(4): 1796-1805.

2. Elkins, J. M., Fedele, V., Szklarz, M., Abdul Azeez, K. R., Salah, E., Mikolajczyk, J., Romanov, S., Sepetov, N., Huang, X. P., Roth, B. L., Al Haj Zen, A., Fourches, D., Muratov, E., Tropsha, A., Morris, J., Teicher, B. A., Kunkel, M., Polley, E., Lackey, K. E., Atkinson, F. L., Overington, J. P., Bamborough, P., Muller, S., Price, D. J., Willson, T. M., Drewry, D. H., Knapp, S. and Zuercher, W. J. (2016). Comprehensive characterization of the Published Kinase Inhibitor Set. Nat Biotechnol 34(1): 95-103.

3. Haslett, C., Guthrie, L. A., Kopaniak, M. M., Johnston, R. B., Jr. and Henson, P. M. (1985). Modulation of multiple neutrophil functions by preparative methods or trace concentrations of bacterial lipopolysaccharide. Am J Pathol 119(1): 101-110.

4. Hidalgo, A., Chilvers, E. R., Summers, C. and Koenderman, L. (2019). The Neutrophil Life Cycle. Trends Immunol 40(7): 584-597.

5. Prince, L. R., Graham, K. J., Connolly, J., Anwar, S., Ridley, R., Sabroe, I., Foster, S. J. and Whyte, M. K. (2012). Staphylococcus aureus induces eosinophil cell death mediated by $\alpha$ hemolysin. PLoS One 7(2): e31506.

6. Rahman, A., Henry, K. M., Herman, K. D., Thompson, A. A., Isles, H. M., Tulotta, C., Sammut, D., Rougeot, J. J., Khoshaein, N., Reese, A. E., Higgins, K., Tabor, C., Sabroe, I., Zuercher, W. J., Savage, C. O., Meijer, A. H., Whyte, M. K., Dockrell, D. H., Renshaw, S. A. and Prince, L. R. (2019). Inhibition of ErbB kinase signalling promotes resolution of neutrophilic inflammation. Elife 8: e50990.

7. Rossi, A. G., Sawatzky, D. A., Walker, A., Ward, C., Sheldrake, T. A., Riley, N. A., Caldicott, A., Martinez-Losa, M., Walker, T. R., Duffin, R., Gray, M., Crescenzi, E., Martin, M. C., Brady, H. J., Savill, J. S., Dransfield, I. and Haslett, C. (2006). Cyclin-dependent kinase inhibitors enhance the resolution of inflammation by promoting inflammatory cell apoptosis. Nat Med 12(9): 10561064.

8. Summers, C., Rankin, S. M., Condliffe, A. M., Singh, N., Peters, A. M. and Chilvers, E. R. (2010). Neutrophil kinetics in health and disease. Trends Immunol 31(8): 318-324.

9. Usher, L. R., Lawson, R. A., Geary, I., Taylor, C. J., Bingle, C. D., Taylor, G. W. and Whyte, M. K. (2002). Induction of neutrophil apoptosis by the Pseudomonas aeruginosa exotoxin pyocyanin: a potential mechanism of persistent infection. $J$ Immunol 168(4): 1861-1868.

10. Webb, P. R., Wang, K. Q., Scheel-Toellner, D., Pongracz, J., Salmon, M. and Lord, J. M. (2000). Regulation of neutrophil apoptosis: a role for protein kinase $\mathrm{C}$ and phosphatidylinositol-3-kinase. Apoptosis 5(5): 451-458. 
11. Wu, P., Nielsen, T. E. and Clausen, M. H. (2015). FDA-approved small-molecule kinase inhibitors. Trends Pharmacol Sci 36(7): 422-439. 